\title{
ATTRACTORS OF MULTIVALUED SEMIFLOWS GENERATED BY DIFFERENTIAL INCLUSIONS AND THEIR APPROXIMATIONS
}

\author{
ALEXEI V. KAPUSTIAN AND JOSÉ VALERO
}

Received 20 October 1999

We prove the existence of global compact attractors for differential inclusions and obtain some results concerning the continuity and upper semicontinuity of the attractors for approximating and perturbed inclusions. Applications are given to a model of regional economic growth.

\section{Introduction}

The theory of multivalued dynamical systems is motivated by differential equations for which it is not known whether the solution corresponding to each initial data is unique or not. In such a case it is not possible to define a semigroup of operators. However, by taking the union of all solutions belonging to a certain class we can define a multivalued semiflow and study in this way the asymptotic behavior of the trajectories. We will recall some results of the abstract theory of attractors for multivalued semiflows developed in $[11,13,14]$ (see also $[3,5])$.

Denote by $X$ a complete metric space with the metric $\rho$ and by $2^{X}(\beta(X)$; $C_{v}(X)$; comp $\left.(X)\right)$ the family of all (nonempty bounded; nonempty, bounded, closed, convex; nonempty compact) subsets of $X$. As usual, $\operatorname{dist}(A, B)=\sup _{y \in A} \inf _{x \in B} \rho(y, x)$ and $\operatorname{dist}_{H}(A, B)=\max \{\operatorname{dist}(A, B)$, $\operatorname{dist}(B, A)\}, A, B \in \beta(X)$, is the Hausdorff metric. Let $B_{\epsilon}(A)=\{y \in X \mid \operatorname{dist}(y, A) \leq \epsilon\}$ be an $\epsilon$-neighborhood of the set $A \subset X$.

A multivalued map $F: X \rightarrow 2^{X}$ is said to be $w$-upper semicontinuous if $\forall x_{0} \in D(F)$, $\forall \epsilon>0, \exists \delta>0$ such that $F(x) \subset B_{\epsilon}\left(F\left(x_{0}\right)\right), \forall x \in B_{\delta}\left(x_{0}\right)$, where $D(F)=\{x \mid F(x) \in$ $P(X)\}$. It is said to be upper semicontinuous if $\forall x_{0} \in D(F)$ and any neighborhood $O\left(F\left(x_{0}\right)\right)$ there exists $\delta>0$ such that $F(x) \subset O\left(F\left(x_{0}\right)\right), \forall x \in B_{\delta}\left(x_{0}\right)$. Obviously, any upper semicontinuous map is $w$-upper semicontinuous, the converse being valid if $F$ has compact values [1, page 45].

A multivalued map $G: \mathbb{R}_{+} \times X \rightarrow P(X)$ is said to be a multivalued semiflow ( $m$-semiflow for short) if $G(0, \cdot)=I d$ and $G\left(t_{1}+t_{2}, x\right) \subset G\left(t_{1}, G\left(t_{2}, x\right)\right), \forall t_{1}, t_{2} \in$ $\mathbb{R}_{+}, \forall x \in X$. The set $\Xi$ is called a global attractor of $G$ if $\Xi \subset G(t, \Xi), \forall t \in \mathbb{R}_{+}$, and 
34 Attractors of multivalued semiflows generated by differential inclusions ...

$\operatorname{dist}(G(t, B), \Xi) \stackrel{t \rightarrow \infty}{\longrightarrow}, \forall B \in \beta(X)$. It is said to be invariant if $\Xi=G(t, \Xi), \forall t \in \mathbb{R}_{+}$. If $\Xi$ is compact then it is the minimal closed set attracting all bounded sets.

The $m$-semiflow $G$ is called point dissipative if there exists $B_{0} \in \beta(X)$ such that $\operatorname{dist}\left(G(t, x), B_{0}\right) \stackrel{t \rightarrow \infty}{\longrightarrow} 0, \forall x \in X$.

Theorem 1.1 (see [14, Theorem 3 and Proposition 1]). Let for any $t \in \mathbb{R}_{+}, G(t, \cdot)$ : $X \rightarrow C(X)$ be upper semicontinuous. Suppose that $G$ is point dissipative and that for some $t_{0}>0$ the operator $G\left(t_{0}, \cdot\right)$ is compact. Then $G$ has the global compact attractor $\Xi$.

Concerning the dependence of attractors on a parameter from the proof of [11, Theorem 4] it follows the following theorem.

THeOREM 1.2. Let $\Lambda$ be a metric space, $\lambda_{0}$ be a non-isolated point and $G_{\lambda}: \mathbb{R}_{+} \times X \rightarrow$ $P(X), \lambda \in \Lambda$, be a family of $m$-semiflows satisfying:

(1) for each $\lambda \in \Lambda, G_{\lambda}$ has a global attractor $\Xi_{\lambda}$ and $\cup_{\lambda \in \Lambda} \Xi_{\lambda} \in \beta(X)$;

(2) the map $\lambda \mapsto G_{\lambda}(t, \Xi), \Xi=\overline{\cup_{\lambda \in \Lambda} \Xi_{\lambda}}$, is w-upper semicontinuous at $\lambda_{0}$ for large $t$.

Then $\operatorname{dist}\left(\Xi_{\lambda}, \Xi_{\lambda_{0}}\right) \rightarrow 0$, as $\lambda \rightarrow \lambda_{0}$.

Other approaches to the problem of non-uniqueness is the construction of the socalled trajectory attractors (see $[8,15,18]$ ) or multivalued semiflows via the nonstandard framework [7].

Whereas in $[4,21]$ are considered differential inclusions generating a semigroup of operators in this paper we study, as in [14], inclusions generating a multivalued semiflow. This paper is organized as follows. In Section 2, we extend the results of [14] on existence of a global compact attractor $\Xi$ for the differential inclusion

$$
\begin{gathered}
\frac{d y}{d t} \in-\partial \phi(y)+F(y), \quad t \in[0 ; T], \\
y(0)=y_{0},
\end{gathered}
$$

where $F: H \rightarrow 2^{H}$ is a multivalued map in a Hilbert space $H$. In Sections 3 and 4, we prove that for a certain class of approximating maps $F_{n}$ of the multivalued righthand side $F$ the corresponding attractors $\Xi_{n}$ converge in the Hausdorff metric to $\Xi$. Finally, in Section 5 we prove the upper semicontinuity of the global attractor under a small perturbation of the map $F, F_{\epsilon}=F+\epsilon S, \epsilon>0$. All these results are applied to boundary value problems and in particular to a model of regional economic growth.

\section{Existence of the global attractor}

Let $H$ be a real separable Hilbert space, $(\cdot, \cdot),\|\cdot\|$ be the scalar product and norm in $H$, respectively, $\phi: H \mapsto(-\infty,+\infty]$ be a proper, convex, lower semicontinuous function and let $\partial \phi: D(\partial \phi) \subset H \mapsto 2^{H}$ be its subdifferential. 
Consider the problem

$$
\begin{gathered}
\frac{d y}{d t} \in-\partial \phi(y)+F(y), \quad t \in[0 ; T], \\
y(0)=y_{0} \in H,
\end{gathered}
$$

where $F: H \rightarrow 2^{H}$ and satisfy the properties:

(G1) $F: H \rightarrow C_{v}(H)$;

(G2) $\exists D_{1}, D_{2} \geq 0$ such that $\sup _{u \in F(v)}\|u\| \leq D_{1}+D_{2}\|v\|, \forall v \in H$;

(G3) $F$ is $w$-upper semicontinuous;

(G4) $\exists \delta>0, M>0$ such that $\forall u \in D(\partial \phi),\|u\|>M, \forall y \in-\partial \phi(u)+F(u)$,

$$
(y, u) \leq-\delta
$$

(G5) $\forall R>0$ the set $M_{R}=\{u \in H \mid\|u\| \leq R, \phi(u) \leq R\}$ is compact in $H$.

Further we denote $X=\overline{D(\phi)}$.

Definition 2.1. The continuous function $y:[0, T] \rightarrow X$ is called an integral solution of problem (2.1) if $y(0)=y_{0}$ and there exists $f \in L_{1}([0, T], X), f(\tau) \in F(y(\tau))$, a.e. $\tau \in(0, T)$, such that $\forall u \in D(\partial \varphi), \forall v \in-\partial \varphi(u)$,

$$
\|y(t)-u\|^{2} \leq\|y(s)-u\|^{2}+2 \int_{s}^{t}(f(\tau)+v, y(\tau)-u) d \tau, \quad t \geq s .
$$

Further we shall denote each integral solution by $y(\cdot)=I\left(y_{0}\right) f(\cdot)$. The integral solution $y(\cdot)$ is called a strong one if it is absolutely continuous on $(0, T)$ and $d y / d t \in$ $-\partial \phi(y(\tau))+f(\tau)$, a.e. on $(0, T)$.

According to [20, Theorem 2.1] $\forall x_{0} \in X, \forall T>0$, there exists an integral solution of (2.1), $x(\cdot)=I\left(x_{0}\right) f(\cdot), x(0)=x_{0}$. Moreover, the set of all integral solutions on $[0, T]$ starting from the point $x_{0}$ (denoted by $\Theta_{F}^{T}\left(x_{0}\right)$ ) is a connected compact set in the space $C(0, T ; X)$ and the map $x \mapsto \Theta_{F}^{T}(x)$ is $w$-upper semicontinuous [20, Theorems 2.1 and 4.3].

LEMma 2.2. Under condition (G2) each integral solution of (2.1) is a strong solution.

Proof. According to [6, page 189] it is sufficient to prove that any selection $f(\cdot) \in$ $F(y(\cdot))$, where $y(\cdot)=I\left(u_{0}\right) f(\cdot)$, belongs to $L_{2}(0, T ; X)$. It follows from $(\mathrm{G} 2)$ that $\|f(t)\| \leq D_{1}+D_{2}\|y(t)\|$, but $y(\cdot) \in C(0, T ; X)$, so that $f(\cdot) \in L_{2}(0, T ; X)$.

Now in the same way as in [14] we define the $m$-semiflow $G: \mathbb{R}_{+} \times X \rightarrow P(X)$, $G\left(t, y_{0}\right)=\left\{y(t) \mid y(\cdot)\right.$ is a strong solution of (2.1), $\left.y(0)=y_{0}\right\}$. Following [14, Lemma 6] we can prove that $G\left(t_{1}+t_{2}, x\right)=G\left(t_{1}, G\left(t_{2}, x\right)\right), \forall x \in X, \forall t_{1}, t_{2} \in \mathbb{R}_{+}$.

THEOREM 2.3. Let (G1)-(G5) hold. Then $G$ has the global compact invariant attractor $\Xi$, which is the minimal closed set attracting all bounded sets. 
Proof. We obtain some properties of $G$. First we prove that $\forall t \geq 0, \forall x \in X, G(t, x)$ is compact in $X$. Indeed, from the fact that $\Theta_{F}^{T}(x)$ is compact in $C(0, T ; X)$ we have that $\forall\left\{y_{n}(\cdot)\right\} \subset \Theta_{F}^{T}(x)$ there exist a subsequence and $y(\cdot) \in \Theta_{F}^{T}(x)$ such as $y_{n} \rightarrow$ $y$ in $C(0, T ; X)$. Hence, $y_{n}(t) \rightarrow y(t), \forall t \in[0, T]$, in $X$. It follows that $G(t, x)$ is compact $\forall t \in[0, T]$. On the other hand, we obtain that $G(t, \cdot): X \rightarrow P(X)$ is upper semicontinuous. Indeed, from the fact that $x \mapsto \Theta_{F}^{T}(x)$ is $w$-upper semicontinuous, we have that $\forall \epsilon>0, \forall x \in X, \exists \delta>0$ such that $\left\|x-x_{0}\right\|<\delta$ implies $\Theta_{F}^{T}(x) \subset B_{\epsilon}\left(\Theta_{F}^{T}\left(x_{0}\right)\right)$, that is, for an arbitrary $y(\cdot) \in \Theta_{F}^{T}(x), \exists y_{0}(\cdot) \in \Theta_{F}^{T}\left(x_{0}\right)$ such that $\max _{t \in[0, T]} \| y(t)-$ $y_{0}(t) \| \leq \epsilon$ and then $\forall t \in[0, T],\left\|y(t)-y_{0}(t)\right\| \leq \epsilon$. Thus $G(t, x) \subset B_{\epsilon}\left(G\left(t, x_{0}\right)\right)$ and by virtue of the compactness of $G(t, x)$ the upper semicontinuity is proved.

Let $B_{0}=\{u \in X \mid\|u\| \leq M+\epsilon\}, \epsilon>0$. We show that $G\left(t, B_{0}\right) \subset B_{0}, \forall t \geq 0$. Let $x_{0} \in B_{0}, x(\cdot) \in \Theta_{F}^{T}\left(x_{0}\right)$ be such that $\exists t>0$ for which $x(t) \notin B_{0}$, that is, $\|x(t)\|>M+\epsilon$. As $x(\cdot)$ is continuous, then there exists $t_{0}$ such that $\left\|x\left(t_{0}\right)\right\|=M+\epsilon,\|x(\tau)\| \geq M+\epsilon$, $\forall \tau \in\left[t_{0}, t\right]$. Therefore, using (G4) and the fact that $x(\cdot)$ is a strong solution of (2.1), in a standard way we obtain that $(1 / 2)(d / d \tau)\|x(\tau)\|^{2} \leq-\delta, \forall \tau \in\left[t_{0}, t\right]$, so that $\|x(t)\|^{2} \leq$ $\left\|x\left(t_{0}\right)\right\|^{2}-2 \delta\left(t-t_{0}\right)$, which is a contradiction. Hence, $G\left(t, B_{0}\right) \subset B_{0}, \forall t \geq 0$. Thus, repeating the proof of [14, Theorem 7], we obtain that $\forall x \in X, \exists t_{x}>0$ such that $G(t, x) \subset B_{0}, \forall t \geq t_{x}$. In the same way we also prove that $G\left(t, B_{N}\right) \subset B_{N}, \forall N>M$, $\forall t \geq 0$, where $B_{N}=\{u \in X \mid\|u\| \leq N\}$. Therefore, $G$ is pointwise dissipative and $\bigcup_{\tau \geq 0} G(\tau, B) \in \beta(X), \forall B \in \beta(X)$.

Now we prove that $G(t, B)$ is precompact in $X$ for any $t>0$ and $B \in \beta(X)$. According to (G5) it is sufficient to prove that $\exists R=R(t, B)$ such that $G(t, B) \subset M_{R}$. First we shall show that the set $M(B, T)=\left\{f(\cdot) \mid y(\cdot)=I\left(y_{0}\right) f(\cdot), y \in \Theta_{F}^{T}\left(y_{0}\right), y_{0} \in\right.$ $B\}$ is bounded in $L_{2}(0, T ; X)$. Indeed, there exists $N$ for which $G(t, B) \subset B_{N}, \forall t \geq 0$, and then $\max _{t \in[0, T]}\|y(t)\| \leq N, \forall y(\cdot) \in \Theta_{F}^{T}(B)$. By virtue of $(\mathrm{G} 2),\|f(t)\| \leq D_{1}+$ $D_{2}\|y(t)\| \leq D_{1}+D_{2} N, \forall f(\cdot) \in M(B, T)$. Thus $M(B, T)$ is bounded in $L_{2}(0, T ; X)$. So, repeating the proof of [14, Theorem 8] we obtain that $\forall t>0, \exists R=R(t, B)$ such that $G(t, B) \subset M_{R}$. Therefore $G(t, B)$ is precompact in $X$.

Hence, it follows from Theorem 1.1 that there exists the global compact attractor $\Xi$. Moreover, by [14, Remarks 5 and 8 ], $\Xi=G(t, \Xi), \forall t \geq 0$, and the minimality property holds.

Remark 2.4. Theorem 2.3 generalizes Theorem 9 from [14], in which $F$ is supposed to be Lipschitz in the multivalued sense.

Consider the application of the previous result to the problem

$$
\begin{gathered}
\frac{\partial y}{\partial t} \in \Delta y+f(y)+h, \quad \text { on } \Omega \times(0, T), \\
\left.y\right|_{\partial \Omega}=0, \\
y(x, 0)=y_{0}(x), \quad x \in \Omega,
\end{gathered}
$$

where $h \in L_{2}(\Omega), \Omega \subset \mathbb{R}^{n}$ is a bounded open domain with smooth boundary $\partial \Omega$ and $f: \mathbb{R} \rightarrow 2^{\mathbb{R}}$ satisfies:

(H1) $f: \mathbb{R} \rightarrow C_{v}(\mathbb{R})$; 
(H2) $\exists D_{1}, D_{2} \geq 0$ such that $\sup _{y \in f(s)}|y| \leq D_{1}+D_{2}|s|, \forall s \in \mathbb{R}$;

(H3) $f$ is $w$-upper semicontinuous;

(H4) $\exists M \geq 0, \alpha>0$ such that $\forall s \in \mathbb{R}, \forall y \in f(s), y s \leq\left(\lambda_{1}-\alpha\right)|s|^{2}+M$, where $\lambda_{1}$ is the first eigenvalue of $-\Delta$ in $H_{0}^{1}(\Omega)$.

To come to problem (2.1), we define $F: H \rightarrow 2^{H}, H=L_{2}(\Omega)$,

$$
F(y)=\{\xi+h \mid \xi \in H, \xi(x) \in f(y(x)) \text { a.e. } x \in \Omega\} .
$$

It is well known that $-\Delta$ is the subdifferential of the proper convex lower semicontinuous function $\phi(u)=\int_{\Omega}(1 / 2)|\nabla u|^{2} d x$ with $D(\phi)=H_{0}^{1}(\Omega)$ and (G5) holds [6].

Proposition 2.5. The map $F$ satisfies $(G 1)-(G 4)$.

Proof. Condition (H4) in a standard way [14, Theorem 10] provides that (G4) holds. The map $f$ has compact values and then it is upper semicontinuous, so that it is measurable [2, Proposition 8.2.1]. Hence, there exists a measurable selection $g(s) \in$ $f(s), s \in \mathbb{R}[2$, Theorem 8.1.3]. Then for any $y \in H, g(y(x))$ is a measurable selection of $f(y(x))$. In view of (H2), we have that $\forall y \in H, \forall(\xi+h) \in F(y),\|\xi+h\| \leq$ $\sqrt{\int_{\Omega}|\xi(x)|^{2} d x}+\|h\| \leq \sqrt{\int_{\Omega}\left(D_{1}+D_{2}|y(x)|\right)^{2} d x}+\|h\| \leq \tilde{D}_{1}+\tilde{D}_{2}\|y\|$, so that $F(y) \neq$ $\emptyset, \forall y \in H$, and (G2) holds. Following [14, Lemma 11] we obtain that $F: H \rightarrow C_{v}(H)$.

Now we prove that if $f: \mathbb{R} \rightarrow C_{v}(\mathbb{R})$ is upper semicontinuous and satisfies (H2) then $F$ is upper semicontinuous on $H$. Since the map $f$ is upper semicontinuous, is upper hemicontinuous [1, page 60]. We prove that $F$ is also hemicontinuous, that is, from $u_{n} \rightarrow u$ in $H$ and $\sigma_{n}(p):=\sigma\left(F\left(u_{n}\right), p\right)=\sup _{v \in F\left(u_{n}\right)}(p, v) \rightarrow \sigma_{0}(p), \forall p \in H$, it follows that $\sigma(F(u), p) \geq \sigma_{0}(p)$. Indeed, $\forall p \in H, \forall n \geq 1 \exists v_{n} \in F\left(u_{n}\right)$ such that $\left(p, v_{n}\right)>\sigma_{n}(p)-1 / n$. Moreover, by virtue of (G2) with accuracy to a subsequence $v_{n} \rightarrow v$ weakly in $H$. Now we can use [16, Chapter 3, Theorem 6], taking $X=$ $Y=\mathbb{R}, p=q=2$. Since $\left(u_{n}(x), v_{n}(x)\right) \in \operatorname{graph}(f)$ for a.e. $x \in \Omega, u_{n} \rightarrow u$ in $H$, $v_{n} \rightarrow v$ weakly in $H$, all the conditions of the mentioned theorem hold and we have $v(x) \in f(u(x))$ for a.e. $x \in \Omega$. Then passing to the limit in the last inequality we have $(p, v) \geq \sigma_{0}(p), v \in F(u)$. Thus, $\sup _{v \in F(u)}(p, v)=\sigma(F(u), p) \geq \sigma_{0}(p)$ and hence $F: H \rightarrow C_{v}(H)$ is hemicontinuous. For arbitrary $u_{0} \in H$ conditions (G1)-(G2) hold, so that $F\left(u_{0}\right)$ is weakly compact and convex in $H$ and hence according to [16, Chapter 3 , Theorem 10] $F$ is upper semicontinuous at $u_{0}$. Therefore, G3 is satisfied.

Now, Theorem 2.3 implies the following theorem.

THEOREM 2.6. Let (H1)-(H4) hold. The semiflow generated by (2.4) has the global compact invariant attractor $\Xi$, which is the minimal closed set attracting all bounded sets.

Example 2.7. A model of regional economic growth.

Consider a closed economy on a bounded domain $\Omega \subset \mathbb{R}^{n}$ and the following variables: $y(x, t)$ is the stock of available capital; $u(x, t)$ is the rate of investment. From the local conservation of capital it follows, as a particular case, that the equation (see 
38 Attractors of multivalued semiflows generated by differential inclusions ...

[17, page 603]):

$$
\begin{gathered}
\frac{\partial y}{\partial t}=\Delta y+\omega(y)+g(y)+u, \quad \text { on } \Omega \times(0, T), \\
\left.y\right|_{\partial \Omega}=0, \\
y(x, 0)=y_{0}(x), \quad x \in \Omega, \\
0 \leq u(x, t) \leq \theta(y(x, t)), \quad \text { on } \Omega \times(0, T),
\end{gathered}
$$

where $-\omega(y), \omega$ being non-decreasing, represents a recursive depreciation of capital and $-g(y)$ is the nonlinear rate of demand. The Dirichlet boundary conditions imply the fact that the economy is closed. We assume that the functions $\omega, g: \mathbb{R} \rightarrow \mathbb{R}$, $\theta: \mathbb{R} \rightarrow \mathbb{R}_{+}$are continuous and have at most linear growth.

Define the multivalued map $f: \mathbb{R} \rightarrow 2^{\mathbb{R}}$,

$$
f(s)=\{\omega(s)+g(s)+\xi \mid 0 \leq \xi \leq \theta(s)\} .
$$

It is straightforward to check that (H1)-(H3) hold. If we assume that

$$
\begin{gathered}
(\omega(s)+g(s)+\theta(s)) s \leq\left(\lambda_{1}-\alpha\right) s^{2}+M, \quad \forall s \geq 0, \\
(\omega(s)+g(s)) s \leq\left(\lambda_{1}-\alpha\right) s^{2}+M, \quad \forall s \leq 0,
\end{gathered}
$$

then (H4) is also satisfied. Therefore, equation (2.6) is a particular case of (2.4) and Theorem 2.6 holds.

\section{Approximation of the attractor}

Now we are interested in the possibility of the approximation of the attractor $\Xi$. For this we assume that the following stronger conditions hold instead of (G2) and (G4):

$(\mathrm{G} 2 *) \exists C>0$ such that $\sup _{u \in F(v)}\|u\| \leq C, \forall v \in H$;

$(\mathrm{G} 4 *) \exists \gamma>0$ such that $(\partial \varphi(y), y) \geq \gamma\|y\|^{2}, \forall y \in D(\partial \varphi)$.

Conditions (G2*), (G4*) imply (G2), (G4). Indeed, for any $\xi \in-\partial \varphi(y)+F(y)$ we have $(\xi, y) \leq-\gamma\|y\|^{2}+\sup _{u \in F(y)}\|u\|\|y\| \leq-\gamma\|y\|^{2}+C\|y\|$. Hence $(\xi, y) \leq$ $\|y\|(-\gamma\|y\|+C)$ and condition (G4) holds for $\delta=M=(1 / \gamma)(C+1)$. Due to condition $\left(\mathrm{G}^{*}\right)$ we can use $\left[20\right.$, Theorem 1.1] and construct the sequence $\left\{F_{n}: H \mapsto C_{v}(H)\right\}$ such that $\forall u \in H, F(u)=\bigcap_{n=1}^{\infty} F_{n}(u), F_{n+1}(u) \subset F_{n}(u), F_{n}$ are locally Lipschitz (in the multivalued sense) and have locally Lipschitz selections and for each $F_{n}$ condition $(\mathrm{G} 2 *)$ holds with the same constant $C$. Moreover, $\operatorname{dist}\left(F_{n}(u), F(u)\right) \rightarrow 0, \forall u \in H$. By $F_{n}$ we construct in the same way as before the $m$-semiflows $G_{n}$, since (G1)-(G4) are satisfied for the maps $F_{n}$. From Theorem 2.3 it follows the existence of the compact global invariant attractor $\Xi_{n}$ for each $G_{n}, n \geq 1$. The maps $F_{n}$ are more regular than $F$, so it is interesting to consider whether the attractors $\Xi_{n}$ converge to $\Xi$ in the Hausdorff metric.

Theorem 3.1. Let (G1), (G2*), (G3), $\left(G 4^{*}\right)$ hold. Then $\operatorname{dist}_{H}\left(\Xi, \Xi_{n}\right) \rightarrow 0$, as $n \rightarrow \infty$.

Proof. We note that $\Xi=G(t, \Xi) \subset G_{n}(t, \Xi) \subset B_{\epsilon}\left(\Xi_{n}\right), \forall \epsilon>0, t \geq T(\epsilon)$, and since the sets $\Xi_{n}$ are compact, we have $\Xi \subset \Xi_{n}, \forall n \geq 1$. Analogously, $\Xi_{n+1} \subset \Xi_{n}$. Hence, 
$\bigcup_{n=1}^{\infty} \Xi_{n} \cup \Xi=\bigcup_{n=1}^{\infty} \Xi_{n}=\Xi_{1}$. We must show that $\forall \epsilon>0, \exists N$ such that $\Xi_{n} \subset B_{\epsilon}(\Xi)$, $\forall n \geq N$. In view of Theorem 1.2 we have to prove that $\bigcup_{n=1}^{\infty} \Xi_{n} \in \beta(H)$ (but we have already shown that such a set is compact) and for large $t$ the next property holds: $\forall \epsilon>0, \exists N$ such that $G_{n}\left(t, \Xi_{1}\right) \subset B_{\epsilon}\left(G\left(t, \Xi_{1}\right)\right), \forall n \geq N$. Now we prove it. On the set $\Lambda=\{n, n \geq 1,+\infty\}$ we introduce the metric $\rho(m, n)=|1 / m-1 / n|(1 / \infty=0)$. Hence $(\Lambda, \rho)$ is a metric compact space. Let $\lambda_{0}:=+\infty$. Now it is sufficient to verify that the map $\Lambda \ni \lambda \mapsto G_{\lambda}\left(t, \Xi_{1}\right)$ is upper semicontinuous at $\lambda_{0}$. Since $G_{\lambda}\left(t, \Xi_{1}\right)$ is compact for any $\lambda \in \Lambda$ (this follows from the fact that the map $G_{\lambda}(t, \cdot)$ is upper semicontinuous and have compact values [1, page 42]), $(\Lambda, \rho)$ is a compact metric space and $G_{\lambda} \subset G_{1}, \forall \lambda \in \Lambda$, it is sufficient to prove that its graph on $\Lambda$ is compact in $\Lambda \times H$ [2, Proposition 1.4.8], that is, the set $D=\left\{(\lambda, u) \mid \lambda \in \Lambda, u \in G_{\lambda}\left(t, \Xi_{1}\right)\right\}$ is compact in $\Lambda \times H$. Let $\left\{\left(\lambda_{n}, u_{n}\right)\right\} \subset D$. Hence $\lambda_{n} \rightarrow \lambda_{0}$ and we have to prove that there exists $u_{1} \in G_{\lambda_{0}}\left(t, \Xi_{1}\right)$ such that $u_{n} \rightarrow u_{1}$ in $H$ (with accuracy to a subsequence). We have $u_{n}=u_{n}(t), u_{n}(\cdot)=I\left(\eta_{n}\right) f_{n}(\cdot), u_{n}(0)=\eta_{n} \in \Xi_{1}$. Hence, there exists $\eta_{0} \in \Xi_{1}$ and a subsequence such that $\eta_{n} \rightarrow \eta_{0}$. We consider $z_{n}(\cdot)=I\left(\eta_{0}\right) f_{n}(\cdot)$. Let $\sigma-L_{1}(0, T ; H)$ be the space $L_{1}(0, T ; H)$ endowed with the weak topology. In view of the inequality $\left\|f_{n}(\tau)\right\| \leq C$, a.e. $\tau \in(0, T)$, for a subsequence $f_{n} \rightarrow f$ in $\sigma-L_{1}(0, T ; H)$. Since $\left\{f_{n}\right\}$ are uniformly integrable and the semigroup $S(t, \cdot)$ generated by $-\partial \phi$ is compact (this follows from (G5) [10, page 1398]), there exist a subsequence $\left\{z_{n}(\cdot)\right\}$ such that $z_{n} \rightarrow z$ in $C(0, T ; H)$ [9, Theorem 2.3]. Hence, $z_{n} \rightarrow z$ in $C(0, T ; H), f_{n} \rightarrow f$ in $\sigma-L_{1}(0, T ; H)$ and $z(\cdot)=I\left(\eta_{0}\right) f(\cdot)\left[19\right.$, Lemma 1.3]. Therefore $\max _{t \in[0, T]} \| u_{n}(t)-$ $z(t)\left\|\leq \max _{t \in[0, T]}\right\| I\left(\eta_{n}\right) f_{n}(t)-I\left(\eta_{0}\right) f_{n}(t)\left\|+\max _{t \in[0, T]}\right\| I\left(\eta_{0}\right) f_{n}(t)-I\left(\eta_{0}\right) f(t) \| \leq$ $\left\|\eta_{n}-\eta_{0}\right\|+\max _{t \in[0, T]}\left\|z_{n}(t)-z(t)\right\| \rightarrow 0, n \rightarrow \infty$. Thus $u_{n}(t) \rightarrow z(t), \forall t \in[0, T]$, $z(0)=\eta_{0} \in \Xi_{1}$. We prove the fact that $f(t) \in F(z(t))$ for a.e. $t \in[0, T]$. First we note that $f_{n}(t) \in F_{n}\left(z_{n}(t)\right)$, a.e. $t \in[0, T]$. We prove that $\exists N$ such that $\forall n \geq N, f(t) \in$ $B_{1 / n}\left(F_{n}(z(t))\right)$, a.e. on $(0, T)$. Indeed, let it not be so. Then $\forall N \geq 1, \exists n \geq N$ such that $f(t) \notin B_{1 / n}\left(F_{n}(z(t))\right)$. On the other hand, from the $w$-semicontinuity and the facts proved above $\forall n \geq 1, \exists m(n) \geq n$ such that $F_{n}\left(z_{k}(t)\right) \subset B_{1 / 2 n}\left(F_{n}(z(t))\right), \forall k \geq m(n)$. So $\bigcup_{k \geq m(n)} F_{n}\left(z_{k}(t)\right) \subset B_{1 / 2 n}\left(F_{n}(z(t))\right)$. As $k \geq m(n) \geq n$, so $\bigcup_{k \geq m(n)} F_{k}\left(z_{k}(t)\right) \subset$ $B_{1 / 2 n}\left(F_{n}(z(t))\right)$. Hence, by virtue of the convexity of $F_{n}(z)$ we have $\overline{c o} \bigcup_{k \geq m(n)} f_{k}(t) \subset$ $B_{1 / n}\left(F_{n}(z(t))\right)$ and therefore $f(t) \notin \overline{\operatorname{co}} \bigcup_{k \geq m(n)} f_{k}(t)$. From [19, Proposition 1.1] we obtain a contradiction. Thus $\forall n \geq N, \exists g_{n} \in F_{n}(z(t))$ such that $\left\|g_{n}-f(t)\right\| \leq$ $1 / n$. Hence $g_{n} \rightarrow f(t)$ in $H$ and from $F_{n+1}(z(t)) \subset F_{n}(z(t))$ it follows that $f(t) \in$ $F_{n}(z(t)), \forall n \geq N$. Thus $f(t) \in F(z(t))$, a.e. on $(0, T)$, and $u_{n}=u_{n}(t) \rightarrow z(t)=u_{1} \in$ $G_{\lambda_{0}}\left(t, \Xi_{1}\right)$.

Remark 3.2. Theorem 3.1 holds for inclusion (2.4) if we assume that $D_{2}=0$ in condition (H2). (G2*) and $\left(\mathrm{G} 4^{*}\right)$ will be satisfied with $C=D_{1}(\mu(\Omega))^{1 / 2}$ and $\gamma=\lambda_{1}$.

\section{Dependence on a parameter}

Now we are interested in the continuous dependence on a parameter. Consider the sequence of problems (2.1) with right-hand sides $F_{n}$ satisfying:

(R1) $F_{n}: H \rightarrow C_{v}(H) ; \forall u \in H, \forall n \geq 1$;

(R2) $F_{n+1}(u) \subset F_{n}(u), \forall n \geq 1$; 
Attractors of multivalued semiflows generated by differential inclusions ...

(R3) $\exists D_{1}, D_{2} \geq 0$ such that $\sup _{v \in F_{1}(u)}\|v\| \leq D_{1}+D_{2}\|u\|, \forall u \in H$;

(R4) $F_{n}$ are $w$-upper semicontinuous $\forall n \geq 1$;

(R5) $\forall u \in H, \bigcap_{n=1}^{\infty} F_{n}(u) \neq \emptyset$ and $F(u)=\bigcap_{n=1}^{\infty} F_{n}(u)$ is $w$-upper semicontinuous;

(R6) $\exists \delta>0, M>0$ such that $\forall u \in D(\partial \phi),\|u\|>M, \forall n \geq 1, \forall y \in-\partial \phi(u)+F_{n}(u)$,

$$
(y, u) \leq-\delta
$$

As before we assume that (G5) holds. Since $F(u) \subset F_{n}(u), F_{n+1}(u) \subset F_{n}(u), \forall u \in$ $H, \forall n \geq 1$, conditions (G1)-(G4) hold for all $F_{n}, F$ (with the same constants $D_{1}, D_{2}$ ). Let $G_{n}, G$ be the semiflows corresponding to $F_{n}, F$. Then in view of Theorem 2.3 there exist the global compact attractors $\Xi_{n}, \Xi$ corresponding to $G_{n}, G$, respectively.

Theorem 4.1. Let (R1)-(R6) and (G5) hold. Then $\operatorname{dist}_{H}\left(\Xi_{n}, \Xi\right) \rightarrow 0$, as $n \rightarrow \infty$.

Proof. As in Theorem 1.2, $\Xi \subset \cdots \subset \Xi_{n+1} \subset \Xi_{n} \subset \cdots \subset \Xi_{1}, \forall n \geq 1$, and the desired result will be obtained if we show that for any sequence $u_{n} \in G_{n}\left(t, \Xi_{1}\right)$ there exists $u_{1} \in G\left(t, \Xi_{1}\right)$ such that $u_{n} \rightarrow u_{1}$ in $H$ (with accuracy to a subsequence). From the proof of Theorem 2.3 it follows that $G\left(t, B_{N}\right) \subset B_{N}, G_{n}\left(t, B_{N}\right) \subset B_{N}, \forall n \geq 1, \forall N>M$. Let $u_{n}=u_{n}(t), u_{n}(\cdot)=I\left(\eta_{n}\right) f_{n}(\cdot), u_{n}(0)=\eta_{n} \in \Xi_{1},\left\|\eta_{n}\right\| \leq N, \forall n \geq 1$, where $N>M$. Then $\max _{t \in[0, T]}\left\|u_{n}(t)\right\| \leq N$. Hence, $\left\|f_{n}(t)\right\| \leq D_{1}+D_{2} N$, a.e. on $(0, T)$, and we can use the same arguments as in the final part of the proof of Theorem 1.2.

Remark 4.2. We note that conditions (R1)-(R5) do not imply that $\operatorname{dist}\left(F_{n}(u), F(u)\right) \rightarrow$ 0 , as $n \rightarrow \infty$.

Proof. Consider the space $H=l_{2}=\left\{y=\left.\left(y_{1}, y_{2}, \ldots\right)\left|\sum_{i=1}^{\infty}\right| y_{i}\right|^{2}<\infty\right\}$ and the sequence of constant maps $F_{n}(u) \equiv Y_{n}=\left\{y \in l_{2} \mid y_{1}=\cdots=y_{n}=0,\|y\| \leq 1\right\}, n \geq 1$. The sets $Y_{n}$ are nonempty, bounded, closed and convex and $F(u)=\cap_{n=1}^{\infty} F_{n}(u)=\{0\}$. It is obvious that the maps $F_{n}, F$ are $w$-upper semicontinuous and satisfy (R2)-(R3) (with $\left.D_{1}=1, D_{2}=0\right)$. We take $\xi_{n}=(\underbrace{n, \ldots, 0}, 1,0, \ldots) \in F_{n}$. Since $\left\|\xi_{n}-0\right\|=1$, we have $\operatorname{dist}\left(F_{n}(u), F(u)\right) \geq 1, \forall n \geq 1$.

Consider the sequence of problems

$$
\begin{gathered}
\frac{\partial y}{\partial t} \in \Delta y+f_{n}(y)+h, \quad \Omega \times(0, T), \\
\left.y\right|_{\partial \Omega}=0, \\
y(x, 0)=y_{0}(x), \quad x \in \Omega,
\end{gathered}
$$

where $h \in L_{2}(\Omega), \Omega \subset \mathbb{R}^{n}$ is a bounded open domain with smooth boundary $\partial \Omega$ and $f_{n}: \mathbb{R} \rightarrow 2^{\mathbb{R}}$ satisfy:

(L1) $f_{n}: \mathbb{R} \rightarrow C_{v}(\mathbb{R}), f_{n+1}(t) \subset f_{n}(t), \forall t \in \mathbb{R}, \forall n \geq 1$;

(L2) $\exists D_{1}, D_{2} \geq 0$ such that $\sup _{y \in f_{1}(s)}|y| \leq D_{1}+D_{2}|s|, \forall s \in \mathbb{R}$; 
(L3) $f_{n}$ are $w$-upper semicontinuous $\forall n \geq 1$;

(L4) $\exists M \geq 0, \alpha>0$ such that $\forall s \in \mathbb{R}, \forall n \geq 1, \forall y \in f_{n}(s), y s \leq\left(\lambda_{1}-\alpha\right)|s|^{2}+M$. Define $F_{n}, F: H \rightarrow 2^{H}, H=L_{2}(\Omega)$,

$$
\begin{gathered}
F_{n}(y)=\left\{\xi+h \mid \xi \in H, \xi(x) \in f_{n}(y(x)) \text { a.e. } x \in \Omega\right\}, \\
F(y)=\left\{\xi+h \mid \xi \in H, \xi(x) \in \cap_{n=1}^{\infty} f_{n}(y(x)) \text { a.e. } x \in \Omega\right\} .
\end{gathered}
$$

Proposition 4.3. The maps $F, F_{n}$ satisfy $(R 1)-(R 6)$.

Proof. Condition (L4) in a standard way [14, Theorem 10] provides that (R6) holds. It follows from (L1)-(L4) and Proposition 2.5 that the maps $F_{n}$ satisfy (R1)-(R4).

(L1)-(L3) imply that all $f_{n}$ are upper semicontinuous (because they are compactvalued) and for any $t \in \mathbb{R}, a>0$, map the ball $B_{a}(t)$ into subsets of some compact set in $\mathbb{R}$. As $\left\{f_{n}(t)\right\}$ is a centered family of compacts, so $\bigcap_{n=1}^{\infty} f_{n}(t) \neq \emptyset$ and in view of [12, page 60] $f(\cdot)=\bigcap_{n=1}^{\infty} f_{n}(\cdot)$ is upper semicontinuous at $t$. It follows now from (L1)-(L4) that $f$ satisfies (H1)-(H4). Then using again Proposition 2.5 we obtain that (R5) holds.

Let $\Xi_{n}, \Xi$ be the global attractors corresponding to $f_{n}, f$, respectively. As a consequence of Theorem 4.1 we have the following theorem.

Theorem 4.4. Let (L1)-(L4) hold. Then $\operatorname{dist}_{H}\left(\Xi_{n}, \Xi\right) \rightarrow 0$, as $n \rightarrow \infty$.

Example 4.5. A model of regional economic growth.

Consider in (2.6) a sequence of functions $\theta_{n}$ such that $\theta_{n+1}(s) \leq \theta_{n}(s), \forall n \geq 1, \forall s \in$ $\mathbb{R}$, and $\theta_{1}$ satisfies (2.8). Then (L1)-(L4) hold and Theorem 4.4 takes place.

\section{Perturbed differential inclusions}

We are now interested in the upper semicontinuity of the global attractor for inclusion (2.1) under small perturbations. Consider the family of differential inclusions

$$
\begin{gathered}
\frac{d u}{d t} \in-\partial \varphi(u)+F(u)+\epsilon S(u), \\
u(0)=u_{0},
\end{gathered}
$$

where $\epsilon \geq 0$ is a small parameter and $S, F: H \rightarrow 2^{H}$ are multivalued maps satisfying (G1)-(G3) and

$(\mathrm{G} 4 * *)$ there exist $\epsilon_{0}>0, \delta>0, M>0$ such that $\forall \epsilon \leq \epsilon_{0}, \forall u \in D(\partial \varphi),\|u\|>M$, $\forall y \in-\partial \varphi(u)+F(u)+\epsilon S(u)$,

$$
(y, u) \leq-\delta
$$

Lemma 5.1. The maps $S_{\epsilon}(u)=F(u)+\epsilon S(u)$ are $w$-upper semicontinuous. 
Proof. Let $\eta>0$ be arbitrary and $\gamma>0$ be such that $\gamma+\epsilon \gamma \leq \eta$. In view of the $w$-upper semicontinuous of $F, S$ there exists $\delta>0$ such that if $\left\|u-u_{0}\right\| \leq \delta$ then

$$
\operatorname{dist}\left(S_{\epsilon}(u), S_{\epsilon}\left(u_{0}\right)\right) \leq \operatorname{dist}\left(F(u), F\left(u_{0}\right)\right)+\epsilon \operatorname{dist}\left(S(u), S\left(u_{0}\right)\right) \leq \eta .
$$

On the other hand, it is evident that $S_{\epsilon}$ satisfy (G1) and (G2) with $D_{1 \epsilon}=\epsilon D_{1}^{S}+D_{1}^{F}$, $D_{2 \epsilon}=\epsilon D_{2}^{S}+D_{2}^{F}$, where $D_{i}^{S}, D_{i}^{F}$ are the constants in condition (G2) corresponding to $S$ and $F$, respectively. If condition (G5) is also satisfied then in view of Theorem 2.3 for each $\epsilon \leq \epsilon_{0}$ inclusion (5.1) generates the multivalued semiflow $G_{\epsilon}: \mathbb{R}_{+} \times \overline{D(\varphi)} \rightarrow$ $\operatorname{Comp}(\overline{D(\varphi)})$ which has the global compact invariant attractor $\Xi_{\epsilon}$.

Define the set-valued map $R(u)=\cup_{0 \leq \epsilon \leq \epsilon_{0}} \epsilon S(u)$.

Lemma 5.2. The map $R$ satisfies $(G 1)-(G 3)$ and $\left(G 4^{* *}\right)$ replacing $\epsilon S$ by $R$.

Proof. It is clear that the set $R(u)$ is nonempty and bounded. Let $y_{n} \in R(u), y_{n} \stackrel{n \rightarrow \infty}{\longrightarrow} y$. Then $y_{n}=\epsilon_{n} z_{n}, z_{n} \in S(u)$. If there exists a subsequence $\epsilon_{n^{\prime}} \rightarrow 0$ then $y=0 \in R(u)$. In another case there exists $n_{0}$ such that $\epsilon_{n} \in\left[\delta, \epsilon_{0}\right], \forall n \geq n_{0}$, for some $\delta>0$. Take a converging subsequence $\epsilon_{n^{\prime}} \rightarrow \epsilon_{1} \in\left[\delta, \epsilon_{0}\right]$. It follows that $z_{n^{\prime}}=y_{n^{\prime}} / \epsilon_{n^{\prime}} \rightarrow y / \epsilon_{1}=z \in$ $S(u)$, since $S(u)$ is closed. Hence, $y=\epsilon_{1} z \in R(u)$, so that $R(u)$ is closed. Further, let $\epsilon y, \epsilon_{1} z \in R(u)$ be arbitrary. Suppose that $\epsilon \leq \epsilon_{1}$. Then for any $\alpha \in[0,1]$,

$$
\alpha \epsilon y+(1-\alpha) \epsilon_{1} z=\epsilon_{2}\left(\alpha^{\prime} y+\left(1-\alpha^{\prime}\right) z\right)=\epsilon_{2} v,
$$

where $\epsilon_{2}=\alpha \epsilon+(1-\alpha) \epsilon_{1}, \alpha^{\prime}=\alpha\left(\epsilon / \epsilon_{2}\right) \in[0,1]$. Since $S(u)$ is convex, $v \in S(u)$ and then $R(u)$ is convex. Therefore, $R(u) \in C_{v}(H)$ and (G1) holds.

Let us check (G3). Let $u$ be arbitrary. Since $S$ is $w$-upper semicontinuous, for any $\gamma>0$ there exists $\delta>0$ such that if $\|u-v\| \leq \delta$, then $S(v) \subset O_{\gamma}(S(u))$. Let $\epsilon y \in R(v)$ be arbitrary. We take $h \in S(u)$ such that $\operatorname{dist}(y, R(u))=\|y-h\|$. Then

$$
\operatorname{dist}(\epsilon y, R(u)) \leq\|\epsilon y-\epsilon h\| \leq \epsilon_{0} \gamma .
$$

It follows that $\operatorname{dist}(R(v), R(u)) \leq \epsilon_{0} \gamma$, if $\|u-v\| \leq \delta$, so that $R$ is $w$-upper semicontinuous.

Finally, it is evident that $R$ satisfies (G2) with $D_{1}^{R}=\epsilon_{0} D_{1}^{S}, D_{2}^{R}=\epsilon_{0} D_{2}^{S}$, and also that $\left(\mathrm{G} 4^{* *}\right)$ holds.

Theorem 5.3. Let the maps $F, S$ satisfy $(G 1)-(G 3),\left(G 4^{* *}\right)$ and $(G 5)$ hold. Then $\operatorname{dist}\left(\Xi_{\epsilon}, \Xi_{0}\right) \rightarrow 0$, as $\epsilon \rightarrow 0^{+}$.

Proof. From Theorem 1.2 it follows that it is sufficient to check that $\cup_{\epsilon \leq \epsilon_{0}} \Xi_{\epsilon} \in$ $\beta(\overline{D(\varphi)})$ and that the map $\epsilon \mapsto G_{\epsilon}\left(t, \overline{\cup_{\epsilon \leq \epsilon_{0}} \Xi_{\epsilon}}\right)$ is $w$-upper semicontinuous at $\epsilon=0$ for any $t \geq 0$.

First, we note that for any $\epsilon \leq \epsilon_{0}, \Xi_{\epsilon}$ belongs to the ball $B^{\alpha}=\{u \in H \mid\|u\| \leq$ $M+\alpha$, where $\alpha>0$. To prove this fact we shall use that for any $\gamma>0$ and $u \in$ $\overline{D(\varphi)}$ there exists $T(u, \epsilon)$ such that $G_{\epsilon}(T, u) \in B^{\gamma}$ and also that $G_{\epsilon}\left(t, B^{\gamma}\right) \subset B^{\gamma}$, 
$\forall t \geq 0, \forall \epsilon \leq \epsilon_{0}$ (see the proof of Theorem 2.3). Let $\gamma<\alpha$. Since $G_{\epsilon}(T, \cdot)$ is upper semicontinuous (see again Theorem 2.3), for any $u \in \Xi_{\epsilon}$ we can find a neighborhood $O(u)$ such that $G_{\epsilon}(T, O(u)) \subset B^{\alpha}$. Since $\Xi_{\epsilon}$ is compact, from the covering $\cup_{u \in \Xi_{\epsilon}} O(u)$ we can obtain a finite subcovering $\cup_{i=1}^{n} O\left(u_{i}\right)$. Hence, $\Xi_{\epsilon} \subset G_{\epsilon}\left(t, \Xi_{\epsilon}\right) \subset B^{\alpha}$ (we take $\left.t \geq \max _{i}\left\{T\left(u_{i}, \epsilon\right)\right\}\right)$, as required. Hence, $\cup_{\epsilon \leq \epsilon_{0}} \Xi_{\epsilon} \in \beta(\overline{D(\varphi)})$.

In order to check the second property we shall prove first that the set $K_{0}=\overline{\cup_{\epsilon \leq \epsilon_{0}} \Xi_{\epsilon}}$ is compact. Let $G_{R}$ be the semiflow generated by inclusion (5.1) if we replace the map $\epsilon S$ by $R$. Since $\epsilon S(u) \subset R(u), \forall \epsilon \leq \epsilon_{0}$, it is clear that $G_{\epsilon}(u) \subset G_{R}(u), \forall u \in \overline{D(\varphi)}$, $\forall \epsilon \leq \epsilon_{0}$. From Theorem 2.3 and Lemma 5.2 it follows that $G_{R}$ has a compact global attractor $\Xi_{R}$. Obviously, $\Xi_{R}$ is a globally attracting set for each $G_{\epsilon}, \epsilon \leq \epsilon_{0}$. Hence, since $\Xi_{\epsilon}$ is the minimal closed set that attracts any bounded set for $G_{\epsilon}$, it follows that $\Xi_{\epsilon} \subset \Xi_{R}, \forall \epsilon \leq \epsilon_{0}$. Therefore, $K_{0}$ is compact.

Suppose that the map $\epsilon \mapsto G_{\epsilon}\left(t, \overline{\cup_{\epsilon \leq \epsilon_{0}} \Xi_{\epsilon}}\right)$ is not $w$-upper semicontinuous at $\epsilon=0$ for some $t>0$. Then there exists a $\gamma$-neighborhood $O_{\gamma}$ of $G_{0}\left(t, K_{0}\right)$ and a sequence $u^{\epsilon_{n}} \in G_{\epsilon_{n}}\left(t, K_{0}\right), \epsilon_{n} \rightarrow 0^{+}$, such that $u^{\epsilon_{n}} \notin O_{\gamma}$. Then $u^{\epsilon_{n}}=u_{\epsilon_{n}}(t)$, where $u_{\epsilon_{n}}(\cdot)=$ $I\left(u_{\epsilon_{n}}^{0}\right) f_{\epsilon_{n}}(\cdot), u_{\epsilon_{n}}^{0} \in K_{0}$, and $f_{\epsilon_{n}}(\tau) \in F\left(u_{\epsilon_{n}}(\tau)\right)+\epsilon_{n} S\left(u_{\epsilon_{n}}(\tau)\right)$, a.e. $\tau \in(0, t)$. Arguing as in Theorems 3.1, 4.1 we obtain the existence of a subsequence (denoted again by $\epsilon_{n}$ ) and functions $f, u$ such that $f_{\epsilon_{n}} \rightarrow f$ in $\sigma-L_{1}([0, t], H), u_{\epsilon_{n}}^{0} \rightarrow u_{0} \in K_{0}, u_{\epsilon_{n}} \rightarrow u$ in $C([0, t], H)$ and $u(\cdot)=I\left(u_{0}\right) f(\cdot)$. We have to prove that $f(\tau) \in F(u(\tau))$, a.e. $\tau \in(0, t)$.

In view of [19, Proposition 1.1] for a.a. $\tau \in(0, t), f(\tau) \in \cap_{m=1}^{\infty} \overline{\mathrm{co}} \cup_{n \geq m} f_{\varepsilon_{n}}(\tau)$. Fix $\tau \in(0, t)$. Since $F$ is $w$-upper semicontinuous and using condition (G2) for the map $S$, we obtain that for any $\delta>0$ there exists $n>0$ such that $\forall k \geq n$,

$$
\begin{aligned}
\operatorname{dist} & \left(F\left(u_{\epsilon_{k}}(\tau)\right)+\epsilon_{k} S\left(u_{\epsilon_{k}}(\tau)\right), F(u(\tau))\right) \\
& \leq \operatorname{dist}\left(F\left(u_{\epsilon_{k}}(\tau)\right)+\epsilon_{k} S\left(u_{\epsilon_{k}}(\tau)\right), F\left(u_{\epsilon_{k}}(\tau)\right)\right)+\operatorname{dist}\left(F\left(u_{\epsilon_{k}}(\tau)\right), F(u(\tau))\right) \\
& \leq \epsilon_{k}\left(D_{1}^{S}+D_{2}^{S}\left\|u_{\epsilon_{k}}(\tau)\right\|\right)+\frac{\delta}{2} \leq \delta .
\end{aligned}
$$

Since $F(u(\tau))$ is convex, this implies that $\overline{c o} \cup_{k \geq n} f_{\varepsilon_{k}}(\tau) \subset O_{\delta}(F(u(\tau)))$. Hence, since $F(u(\tau))$ is closed, $f(\tau) \in F(u(\tau))$, a.e. $t \in(0, t)$. Then $u^{\varepsilon_{n}} \rightarrow u(t) \in G_{0}\left(t, K_{0}\right)$, which is a contradiction.

Consider now the family of boundary value problems

$$
\begin{gathered}
\frac{\partial u}{\partial t} \in \Delta u+f(u)+\epsilon j(u)+h, \quad \text { on } \Omega \times(0, T), \\
\left.u\right|_{\partial \Omega}=0, \\
u(0)=u_{0},
\end{gathered}
$$

where $h \in L_{2}(\Omega), \epsilon \geq 0$ is small, $f, j: \mathbb{R} \rightarrow 2^{\mathbb{R}}$ satisfy (H1)-(H3) and $f$ satisfies (H4).

Define the maps $F, S: H \rightarrow 2^{H}, H=L_{2}(\Omega)$, by

$$
\begin{aligned}
& F(u)=\{y \in H \mid y(x) \in f(u(x))+h(x), \text { a.e. on } \Omega\}, \\
& S(u)=\{y \in H \mid y(x) \in j(u(x)), \text { a.e. on } \Omega\} .
\end{aligned}
$$


44 Attractors of multivalued semiflows generated by differential inclusions ...

It follows from Proposition 2.5 that the maps $F, S$ satisfy (G1)-(G3).

Lemma 5.4. Condition $\left(G 4^{* *}\right)$ holds.

Proof. Since $f$ satisfies (H4) and (G2) holds for $S$, we have that $\forall u \in D(\partial \varphi), \forall y \in$ $-\partial \varphi(u)+F(u)+\epsilon S(u)$,

$$
\begin{aligned}
(y, u) & \leq-\lambda_{1}\|u\|^{2}+\left(\lambda_{1}-\alpha\right)\|u\|^{2}+M \mu(\Omega)+\epsilon\left(D_{1}+D_{2}\|u\|\right)\|u\|+\|u\|\|h\| \\
& \leq\left(-\frac{\alpha}{2}+\epsilon D_{2}\right)\|u\|^{2}+M \mu(\Omega)+\frac{\epsilon^{2} D_{1}^{2}}{\alpha}+\frac{1}{\alpha}\|h\|^{2} .
\end{aligned}
$$

Taking $\epsilon_{0}=\alpha / 4 D_{2}$ the last inequality implies that condition $\left(\mathrm{G} 4^{* *}\right)$ holds.

Since (G5) is also satisfied, we have obtained a particular case of inclusion (5.1), so that Theorem 5.3 implies the following result.

Theorem 5.5. Let $f, j$ satisfy $(H 1)-(H 3)$ and $f$ satisfy $(H 4)$. Then $\operatorname{dist}\left(\Xi_{\epsilon}, \Xi_{0}\right) \rightarrow 0$, as $\epsilon \rightarrow 0^{+}$.

Example 5.6. A model of regional economic growth.

Consider in (2.6) the family of functions $g_{\epsilon}=g_{1}+\epsilon g_{2}, \theta_{\epsilon}=\theta_{1}+\epsilon \theta_{2}$, where $g_{1}, \theta_{1}$ satisfy the same conditions as $g, \theta$ and $g_{2}, \theta_{2}$ are continuous and have at most linear growth. Define the multivalued maps $f, j: \mathbb{R} \rightarrow 2^{\mathbb{R}}$,

$$
\begin{aligned}
f(s) & =\left\{\omega(s)+g_{1}(s)+\xi \mid 0 \leq \xi \leq \theta_{1}(s)\right\}, \\
j(s) & =\left\{g_{2}(s)+\xi \mid 0 \leq \xi \leq \theta_{2}(s)\right\} .
\end{aligned}
$$

Then we obtain a particular case of inclusion (5.7), so that Theorem 5.5 holds.

Finally, we remark that if in problems (2.4), (4.2), and (5.7) we replace the operator $-\Delta$ by $A(u)=-\sum_{i=1}^{n}\left(\partial / \partial x_{i}\right)\left(\left|\partial y / \partial x_{i}\right|^{p-2}\left(\partial y / \partial x_{i}\right)\right), p>2$, then all the results remain valid. In this case, conditions (H4), (L4) are not necessary. Indeed, we prove that $\left(\mathrm{G} 4{ }^{* *}\right)$ holds $((\mathrm{G} 4)$ and $(\mathrm{R} 6)$ can be proved in a similar way). It follows from Poincaré inequality that $\langle A u, u\rangle=\|\nabla u\|_{L_{p}}^{p} \geq D\|u\|_{L_{p}}^{p}$ for some $D>0$. Let $\epsilon_{0}>0$ be arbitrary but fixed. Then using the Young inequality we have that $\forall u \in D(\partial \varphi), \forall \epsilon \leq \epsilon_{0}$, $\forall y \in-A(u)+F(u)+\epsilon S(u)$,

$$
\begin{aligned}
(y, u) & \leq-D\|u\|_{L_{p}}^{p}+\epsilon\left(D_{1}+D_{2}\|u\|\right)\|u\|+\left(D_{3}+D_{4}\|u\|\right)\|u\|+\|u\|\|h\| \\
& \leq-\widetilde{D}\|u\|^{p}+K
\end{aligned}
$$

where $\widetilde{D}>0$, so that $\left(\mathrm{G}^{* *}\right)$ holds.

\section{Acknowledgement}

The second author has been supported by PB-2-FS-97 grant (Fundación Séneca de la Comunidad Autónoma de Murcia). 


\section{References}

[1] J.-P. Aubin and A. Cellina, Differential Inclusions. Set-valued Maps and Viability Theory, Fundamental Principles of Mathematical Sciences, vol. 264, Springer-Verlag, Berlin, 1984. MR 85j:49010. Zbl 538.34007.

[2] J.-P. Aubin and H. Frankowska, Set-valued Analysis, Systems and Control: Foundations and Applications, vol. 2, Birkhäuser Boston, Boston, 1990. MR 91d:49001. Zbl 713.49021.

[3] A. V. Babin, Attractor of the generalized semigroup generated by an elliptical equation in a cylindrical domain, Russian Acad. Sci. Izv. Math. 44 (1995), no. 2, 207-233, translated from Izv. Ross. Akad. Nauk Ser. Mat. 58 (1994), no. 2, 3-18. Zbl 839.35036.

[4] F. Balibrea and J. Valero, On dimension of attractors of differential inclusions and reaction-diffusion equations, Discrete Contin. Dynam. Systems 5 (1999), no. 3, 515528. MR 2000k:37122. Zbl 991.51555.

[5] J. M. Ball, Continuity properties and global attractors of generalized semiflows and the Navier-Stokes equations, J. Nonlinear Sci. 7 (1997), no. 5, 475-502. MR 98j:58071a. Zbl 903.58020.

[6] V. Barbu, Nonlinear Semigroups and Differential Equations in Banach Spaces, Editura Academiei Republicii Socialiste România, Bucharest; Noordhoff International Publishing, Leiden, 1976, translated from the Romanian. MR 52\#11666. Zbl 328.47035.

[7] M. Capiński and N. J. Cutland, Attractors for three-dimensional Navier-Stokes equations, Proc. Roy. Soc. London Ser. A 453 (1997), no. 1966, 2413-2426. MR 98i:35140. Zbl 990.73265.

[8] V. V. Chepyzhov and M. I. Vishik, Evolution equations and their trajectory attractors, J. Math. Pures Appl. (9) 76 (1997), no. 10, 913-964. MR 99a:34165. Zbl 896.35032.

[9] S. Gutman, Existence theorems for nonlinear evolution equations, Nonlinear Anal. 11 (1987), no. 10, 1193-1206. MR 89d:47142. Zbl 642.47055.

[10] A. Haraux, Attractors of asymptotically compact processes and applications to nonlinear partial differential equations, Comm. Partial Differential Equations 13 (1988), no. 11, 1383-1414. MR 89j:58072. Zbl 676.35008.

[11] A. V. Kapustyan and V. S. Mel'nik, Global attractors of multivalued semidynamical systems and their approximations, Kibernet. Sistem. Anal. (1998), no. 5, 102-111, 189, [translation in Cybernet. Systems Anal. 34 (1998), no. 5, 719-725. Zbl 991.17103]. MR 2000h:37016.

[12] K. Kuratowski, Topology. Vol. II, Academic Press, New York; Państwowe Wydawnictwo Naukowe Polish Scientific Publishers, Warsaw, 1968, New edition, revised and augmented, translated from French by A. Kirkor. MR $41 \# 4467$.

[13] V. S. Mel'nik, Multivalued Dynamics of Nonlinear Infinite-dimensional Systems, Preprint, no. 94-17, Natsional'naya Akademiya Nauk Ukrainy, Institut Kibernetiki im. V. M. Glushkova, Kiev, 1994 (Russian). MR 97h:34086.

[14] V. S. Mel'nik and J. Valero, On attractors of multivalued semi-flows and differential inclusions, Set-Valued Anal. 6 (1998), no. 1, 83-111. MR 99e:34087. Zbl 915.58063.

[15] D. E. Norman, Chemically reacting fluid flows: weak solutions and global attractors, J. Differential Equations 152 (1999), no. 1, 75-135. MR 2000g:35213. Zbl 936.35133.

[16] Z.-P. Oben and I. Èkland, Applied Nonlinear Analysis, "Mir", Moscow, 1988 (Russian), translated from English by B. S. Darkhovskiı̌ and G. G. Magaril-Il'yaev. With a preface by V. M. Tikhomirov. MR 89g:58001.

[17] N. S. Papageorgiou, Evolution inclusions involving a difference term of subdifferentials and applications, Indian J. Pure Appl. Math. 28 (1997), no. 5, 575-610. MR 98j:34020. Zbl 881.34073.

[18] G. R. Sell, Global attractors for the three-dimensional Navier-Stokes equations, J. Dynam. Differential Equations 8 (1996), no. 1, 1-33. MR 98e:35127. Zbl 855.35100. 
46 Attractors of multivalued semiflows generated by differential inclusions ...

[19] A. A. Tolstonogov, Solutions of evolution inclusions. I, Siberian Math. J. 33 (1992), no. 3, 500-511, [translation from Sibirsk. Mat. Zh. 33 (1992), no. 3, 161-174. MR 93g:34026]. Zbl 787.34052 .

[20] A. A. Tolstonogov and Y. I. Umanski1̌, On solutions of evolution inclusions. II, Siberian Math. J. 33 (1992), no. 4, 693-702, [translation from Sibirsk. Mat. Zh. 33 (1992), no. 4, 163-174. MR 93h:34027]. Zbl 791.34016.

[21] J. Valero, On locally compact attractors of dynamical systems, J. Math. Anal. Appl. 237 (1999), no. 1, 43-54. MR 2000i:37144. Zbl 935.37050.

Alexei V. Kapustian: Kiev University Taras Shevchenko, Department of Mechanics and Mathematics, Vladimirskaya, 60, Kiev, Ukraine

José Valero: Universidad Cardenal Herrera, C/Comisario, 3, 03203 Elche (Alicante), SPAIN 


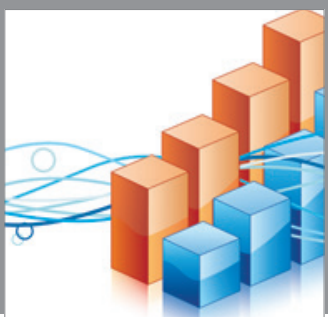

Advances in

Operations Research

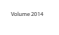

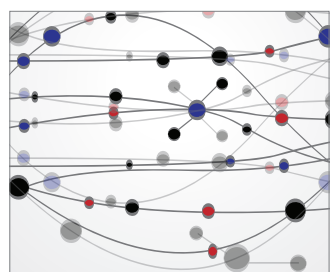

\section{The Scientific} World Journal
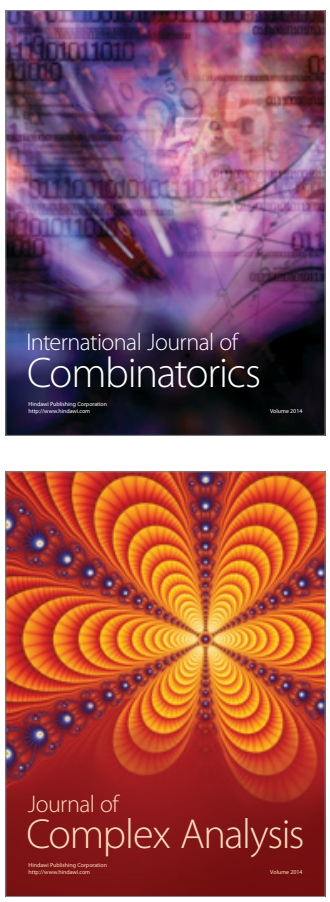

International Journal of

Mathematics and

Mathematical

Sciences
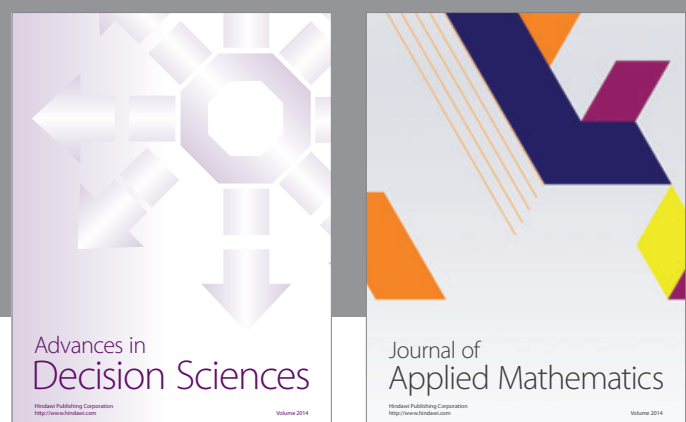

Journal of

Applied Mathematics
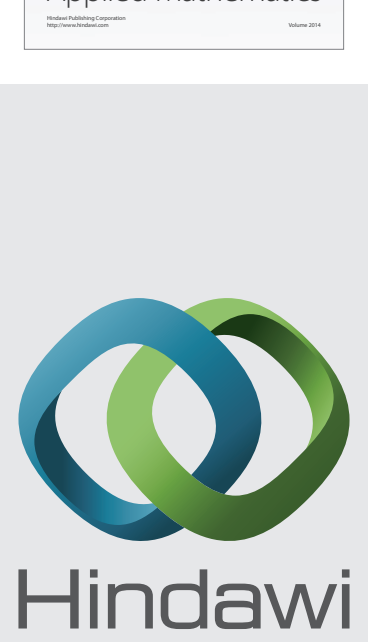

Submit your manuscripts at http://www.hindawi.com
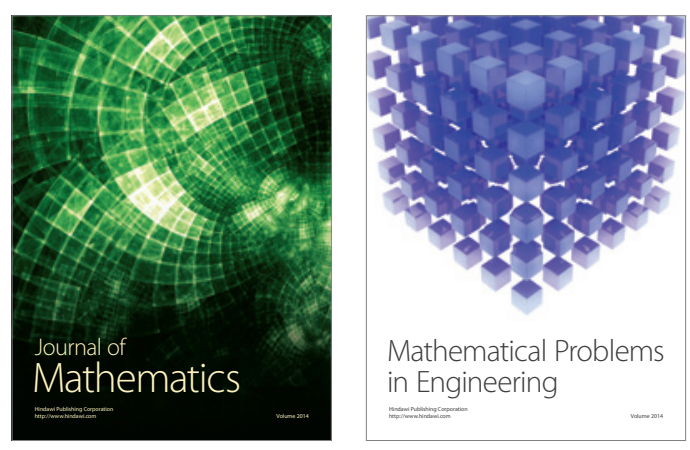

Mathematical Problems in Engineering
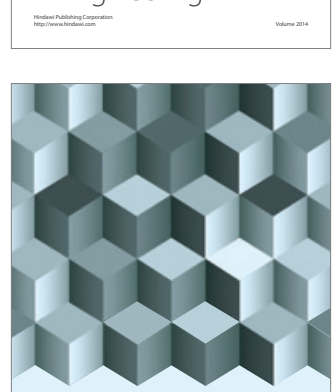

Journal of

Function Spaces
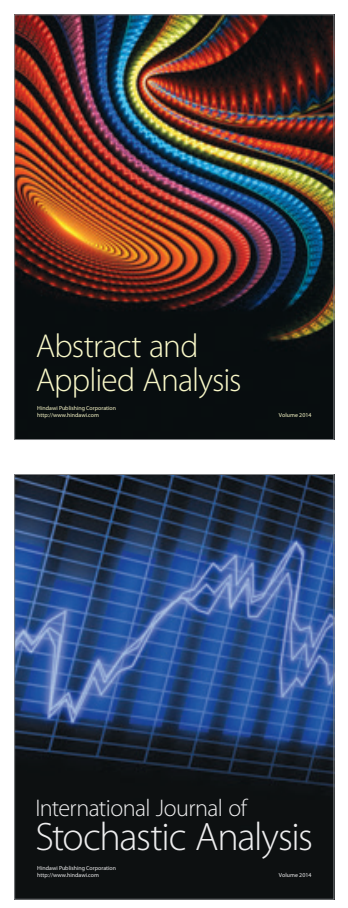

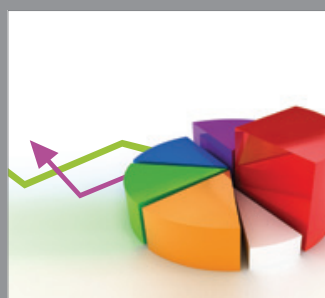

ournal of

Probability and Statistics

Promensencen
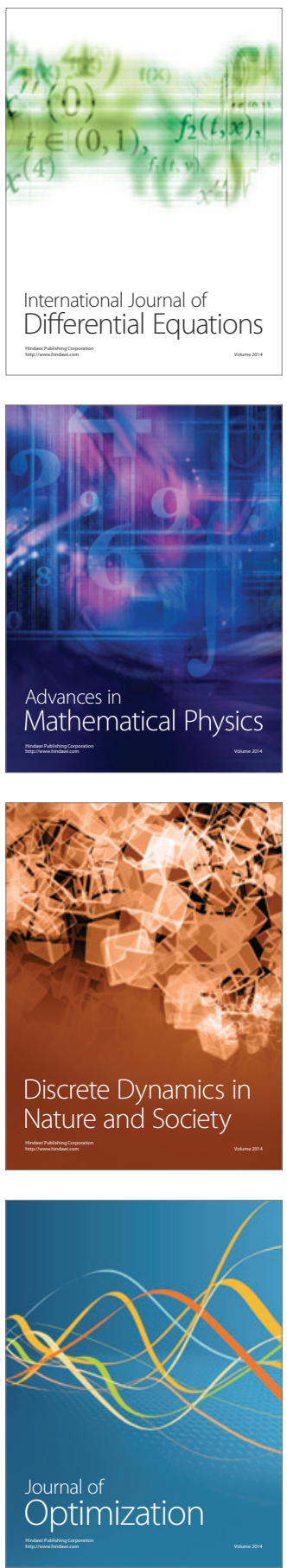